Recepción: 22 / 02 / 2018

Aceptación: 14 / 04 / 2018

Publicación: 15 / 09 / 2018

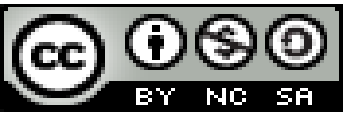

Ciencias de la salud

Artículo de investigación

\title{
Caries dental y maloclusiones en infantes con labio y/o paladar hendido
}

\section{Dental caries and maloclusions in infants with lip and / or sandy palate}

\section{Cárie dentária e maloclusões em lesões com lábio e / ou sandy palate}

\author{
Juan C. Giusti-Barreto I \\ juancarlosg@hotmail.com \\ Gema M. Panchana-Moreira II \\ gemapanchana@gmail.com \\ Tita L. García-Muñoz III \\ titagarcia@gmail.com \\ Guillermina E. Zurita-Yong VI \\ guillerminazurita@gmail.com
}

\section{Correspondencia: juancarlosg@hotmail.com}

I Odontólogo, Magister en Ciencias: Mención Operatoria Dental y Endodoncia, Coordinador Académico de Máster de Endodoncia, Universidad de Carabobo, Venezuela.

II Odontóloga, Residente del Posgrado de Odontopediatria, Universidad de Carabobo, Venezuela.

III Odontóloga, Residente del Posgrado de Odontopediatria, Universidad de Carabobo, Venezuela.

VI Odontóloga, Residente del Posgrado de Odontopediatria, Universidad de Carabobo, Venezuela. 


\title{
Resumen
}

Una de las anomalías craneofacial más comunes es la del labio y/o paladar hendido, generando como una de la patología más común, entre estos pacientes es la caries. Con base, en lo expuesto, se propuso en este artículo evaluar la prevalencia de caries dental y maloclusiones en pacientes con labio y/o paladar hendido. La metodología fue de tipo cuantitativa y descriptivo, con un diseño de corte bibliográfico documental. La población la constituyeron 22 historias clínicas de pacientes con la patología de labio y/o paladar hendido, que reposan en los Servicios de Odontopediatria del hospital Vozandes en la ciudad de Quito, Ecuador. El análisis de la información y datos obtenidos, posterior a la revisión de las historias clínicas, determino que el 93\% de los pacientes presentaban caries dental y el $92 \%$ presento alguna maloclusion. Entre sus conclusiones, se pudo determinar que existe una relación altamente significativa de dependencia entre la caries dental y los pacientes con alguna maloclusion en pacientes con labio y/o paladar hendido.

Palabras claves: labio y/o paladar hendido; caries dental; maloclusiones y odontopediatria.

\begin{abstract}
One of the most common craniofacial anomalies is the lip and / or cleft palate, generating as one of the most common pathology, among these patients is caries. Based on the above, it was proposed in this article to evaluate the prevalence of dental caries and malocclusions in patients with cleft lip and / or palate. The methodology was quantitative and descriptive, with a documentary bibliographic cut design. The population was made up of 22 clinical histories of patients with cleft lip and / or palate pathology, who reside in the Odontopediatria Services of the Vozandes Hospital in the city of Quito, Ecuador. The analysis of the information and data obtained, after the review of the medical records, determined that $93 \%$ of the patients had dental caries and $92 \%$ presented some malocclusion. Among its conclusions, it was determined that there is a highly significant relationship of dependence between dental caries and patients with some malocclusion in patients with cleft lip and / or palate.
\end{abstract}

Keywords: lip and / or cleft palate; dental caries; malocclusions and odontopediatria. 


\section{Resumo}

Uma das anomalias craniofaciais mais comuns é o lábio e / ou fenda palatina, gerando como uma das patologias mais comuns, dentre esses pacientes, a cárie. Com base no exposto, foi proposto neste artigo avaliar a prevalência de cárie dentária e maloclusões em pacientes com fissura labial e / ou palatina. A metodologia foi quantitativa e descritiva, com um desenho de corte bibliográfico documentário. A população foi composta por 22 histórias clínicas de pacientes com patologia labial e / ou palatina, que residem nos Serviços de Odontopediatria do Hospital Vozandes, na cidade de Quito, Equador. A análise das informações e dados obtidos, após a revisão dos prontuários, determinou que $93 \%$ dos pacientes apresentavam cárie dentária e $92 \%$ apresentavam alguma má oclusão. Entre suas conclusões, determinou-se que existe uma relação altamente significativa de dependência entre cárie dentária e pacientes com algumas más oclusões em pacientes com fissura labial e / ou palatina

Palavras chave: lábio e / ou fenda palatina; cárie dentária; maloclusões e odontopediatria.

\section{Introducción}

El labio y paladar hendido es una de las malformaciones congénitas más comunes que afectan el desarrollo de las estructuras bucofaciales y traen como consecuencia al paciente, múltiples problemas en la alimentación, la respiración nasal, la audición, el crecimiento facial, el desarrollo dental, la fonación y la estética. También constituyen un problema biológico y psicológico importante que repercute en el núcleo familiar y en el entorno social. Se constituyen como deficiencias estructurales congénitas a causa de la falta de unión entre algunos procesos faciales embrionarios en formación.

Estas afectan el complejo cráneofacial y son el resultado de la falta de unión entre los procesos frontonasales, maxilar y medial nasal, y laterales; que ocurre entre la sexta y la octava semana de gestación (Mejía y Suárez, 2012). El estudio del labio y/o paladar hendido (LPH), ha sido el objetivo de numerosas investigaciones por tratarse de la anomalía craneofacial congénita más común a nivel mundial; afectando a 1 de cada 500 a 700 recién nacidos, según datos de la Organización Mundial de la Salud (OMS, 2007). 
Según el Estudio Colaborativo Latinoamericano de Malformaciones Congénitas (ECLAMC), la tasa global para labio y paladar hendido es de $10.49 \%$ por $10.000 \mathrm{nv}$, siendo alta en países como Bolivia (25.0:10000), Chile (17.8), Paraguay (15.5) y Ecuador (14.2) y baja en Uruguay (12.2), Perú (8.7) y Venezuela (11.0) (5). La dentición primaria en los niños con labio y paladar hendido es un componente fundamental para la función oral, el desarrollo del habla, el mantenimiento del espacio para la dentición permanente y el éxito del tratamiento de maloclusión que se haga necesario, sin embargo, el logro de una óptima salud bucal puede ser difícil, debido a que estos niños presentan características bucales particulares que pueden influir: anatomía de la zona de la hendidura, anomalías dentales de forma, estructura, número y posición, alteraciones en la erupción dental y fibrosis cicatricial secundaria a la cirugía de labio que causa inmovilidad del labio, afectando la correcta higiene oral y favoreciendo la acumulación de placa dental.

Asimismo, la prevalencia de las hendiduras orales indica Mejía y Suarez (2012), ha sido relacionada con el número de gestaciones de la madre, siendo más prevalente en los casos donde la madre es multípara. Además, se ha observado una relación con la edad materna, y con los antecedentes familiares ya que es más frecuente en los sujetos que tienen historia familiar de hacer sufrido el evento.

Estudios indican, que el niño con LPH es susceptible a una serie de complicaciones que afectan su desarrollo. Entre los signos y síntomas característicos en estos pacientes se encuentran: deficiencia de peso, incompatibilidad labial y del velo faríngeo, trastornos de la succión y deglución, maloclusión, problemas de fonación, respiración oral; y a nivel dental ocurren con mayor frecuencia hipoplasias del esmalte, caries dental, anodoncia, erupción ectópica, gingivitis y periodontitis. Por otro lado, Bedón y Villota (2012), indican que este tipo de pacientes pueden presentar defectos funcionales intraorales como frenillo corto, torus, anquiloglosia, entre otros.

Hasslöf y Twetman (2007), indican que es poca la información e investigación sobre la experiencia de caries en niños con hendiduras de labio y/o paladar hendido, que la revisión sistemática de la literatura no ha podido encontrar evidencia firme para el supuesto de que estos niños tengan una mayor prevalencia de caries dentales, lo que hace necesario evaluar el estado de la dentición primaria y la necesidad de tratamiento de los niños con esta condición médica. Por lo que el objetivo de este artículo se centró en evaluar la prevalencia de caries dental y maloclusiones en pacientes con labio y/o paladar hendido. 


\section{Desarrollo}

Según la Patología Oral y Maxilofacial Contemporánea de Philip, Lewis y Wysocki (2012), el labio fisurado se define "como un trastorno del desarrollo, caracterizado por un defecto en forma de cuña que es consecuencia de una ausencia de fusión de las dos partes del labio en una sola estructura" y el paladar fisurado "como un defecto del desarrollo del paladar caracterizado por la ausencia de fusión completa de las dos crestas palatinas, produciéndose una comunicación con la cavidad nasal"'(pag. 77). El labio y/o paladar hendido (LPH) es una de las malformaciones congénitas estructurales más comunes; afecta el complejo cráneo facial y es el resultado de la falta de unión entre los procesos frontonasal, maxilar y medial nasal, y lateral. Las fisuras unilaterales ocurren cuando la prominencia del lado afectado falla en unirse con la prominencia nasal medial en emergencia. El LPH se puede encontrar de cuatro formas, principalmente: Labio Fisurado (LF). Paladar Fisurado (PF). Labio y paladar hendido unilaterales (LPHu) y Labio y paladar hendido bilateral (LPHb).

\section{Caries dental en pacientes con LPH}

En el mundo, los reportes sobre experiencia de caries dental en niños con LPH son controversiales. Algunos no encuentran diferencias en la prevalencia de experiencia de caries dental (COP-d); en el COP-d promedio entre niños con fisura y sin este, ni entre los diferentes tipos de fisuras $(\mathrm{p}=0,67)$. Por otra parte, algunos reportes han encontrado diferencias, ya sea en la prevalencia de la experiencia de caries dental en dientes primarios, que es significativamente menor para algunos en los niños con fisura comparados con un grupo control, o mayor para este grupo, como lo muestra Al-Dajani (2009), o presentan un mayor porcentaje de dientes anteriores afectados en hendidura bilateral frente a la hendidura unilateral. Otros reportan una mayor prevalencia en niños con hendidura, sin diferencias entre los tipos de hendidura, pero con más superficies cariadas y obturadas a los 5,5 años en LPH.

\section{Maloclusiones en pacientes con LPH}

Los problemas de crecimiento del complejo dentofacial en pacientes con hendidura labial y palatina se reflejan generalmente en la relación dental anteroposterior y transversal. Se han utilizado diferentes métodos de registro de estas relaciones dentales para documentar el resultado de la cirugía en pacientes con fisuras. La mayoría de los métodos de puntuación se basan en la 
presencia de mordida cruzada. Respecto a las maloclusiones más comunes, como características principales de los pacientes con hendidura labial y/o palatina, Vettore y col (2011), en su estudio titulado "Características de maloclusión en pacientes con hendidura labial y/o palatina, publicado en el Journal Europeo de Ortodoncia, enlistan las siguientes, tomadas de una muestra brasileña de 56 pacientes de 6-12 años: relación molar clase II de Angle, el 58.9\%, clase III de Angle, 23.2\%, mordida cruzada anterior $60.7 \%$, mordida cruzada posterior el $39.3 \%$, apiñamiento superior anterior $69.6 \%$, apiñamiento anterior inferior $66.1 \%$. Con respecto al tipo de hendidura palatina y su asociación con el tipo de maloclusión, los autores dividieron la muestra en 4 tipos de hendidura: anterior al foramen incisivo, que pase el foramen incisivo, posterior al foramen incisivo y hendiduras raras.

\section{Metodología}

La metodología fue de tipo cuantitativa y descriptivo, con un diseño de corte bibliográfico documental. La población la constituyeron 22 historias clínicas de pacientes con la patología de labio y/o paladar hendido, que reposan en los Servicios de Odontopediatria del hospital Vozandes en la ciudad de Quito, Ecuador. La información se obtuvo posterior a la revisión de las historias clínicas para lao cual se utilizó un instrumento tipo lista de cotejo que permitió asentar cada una de las características necesarias para el estudio, entre estas edad, sexo y presencia/ausencia de caries dental, enfermedad periodontal y maloclusión. Para la recolección de los datos se empleó el método de observación indirecta a través de la revisión de las historias clínicas seleccionadas para la muestra, y en un instrumento debidamente diseñado para tal fin se registraron los datos relacionados con: número de historia, edad, sexo y presencia/ausencia de caries dental, enfermedad periodontal y maloclusión (Según el odontodiagrama y el diagnóstico clínico realizado por el odontólogo que realizó la evaluación clínica). El análisis descriptivo de los datos se realizó a través del paquete estadístico SPSS versión 23.0.

\section{Análisis y discusión de los resultados}

La edad promedio de los pacientes que asisten al servicio de odontopediatria del hospital Vozandes, en la ciudad de Quito, fue entre 7 a 15 años, presentando un mayor porcentaje del 58\% del sexo masculino. 
En el 93\% del total de los pacientes investigados, presentaban caries dentales; por otro lado, fue posible determinar que las enfermedades periodontales se presentaron en el $65 \%$ de los pacientes sanos. Asimismo, el $92 \%$ presento alguna mal oclusión. En relación a la caries dental, actualmente indican los estudios que la prevalencia de caries en poblaciones de pacientes con LPH, es significativa en este tipo de pacientes.

Si consideramos que la fisura labiopalatina es una de las malformaciones congénitas más frecuentes en el mundo, los pacientes con LPH son susceptibles a una serie de complicaciones que afectan su desarrollo, específicamente plantean Mejías y Suarez (2012), de trastornos de la succión y deglución, maloclusión, problemas de fonación; y a nivel dental ocurren con mayor frecuencia caries dental, gingivitis y periodontitis.

Por su parte, Zhu et al (2010), explica que se viene dando un incremento en la prevalencia de caries dental en niños con LPH, al indicar en su investigación en que existe una prevalencia de caries dental de 70,9\% para la edad de 3 a 5 años; y de 82,3\% para la edad de 6 a 12 años.

Los pacientes con LPH presentaron un mayor recuento de microorganismos asociados a caries, mayor índice de placa bacteriana, sangrado gingival y mayores tiempos de autolimpieza con la consecuente fermentación de azucares y almidones; lo que podría evidenciar la relación entre estos factores y la mayor prevalencia de caries dental y enfermedad periodontal (Quirynenm et al., 2003).

La mayor alteración en los pacientes con LPH, son las maloclusiones; pues al no darse la fusión de los procesos faciales embrionarios, el maxilar superior no se desarrolla de manera adecuada, alterándose la erupción dentaria, lo que conlleva a variaciones en la oclusión (Mejía y Suárez, 2012).

\section{Conclusiones}

Se determinó que existe una relación altamente significativa de dependencia entre la caries dental y los pacientes con alguna maloclusion en pacientes con labio y/o paladar hendido. 
Se constató que los pacientes con labio y/o paladar hendido tienen una alta prevalencia de caries dental, enfermedad periodontal y maloclusiones.

Fue posible explicar que la fisura labiopalatina es una de las malformaciones congénitas más susceptibles a una serie de complicaciones que afectan el normal desarrollo de las funciones de succión y deglución, maloclusión, problemas de fonación; y a nivel dental ocurren con mayor frecuencia caries dental, gingivitis y periodontitis.

\section{Referencias Bibliográficas}

Al-Dajani M. (2011). Comparison of dental caries prevalence in patients with cleft lip and/or palate and their sibling controls. Cleft Palate Craniofac J. 2009; 46(5): 529-31.

Bedón M, Villota G. (2012). Labio y paladar hendido. Tendencias actuales en el manejo exitoso. Archivos de medicina. 12(1):107-119. Clark JD, Mossey PA, Sharp.

Hasslöf P, Twetman S. La prevalencia de caries en los niños con labio leporino y paladar hendido - una revisión sistemática de estudios de casos y controles. Int J Dent Paediatr [seriada en línea] 2007; 17 (5):313-9. Disponible en: http://www.ncbi.nlm.nih.gov/pubmed/17683319

Mejía AC, Suárez D. (2012). Factores de riesgo materno predominantes asociados con labio leporino y paladar hendido en los recién nacidos. Arch Inv. Mat Inf. 4(2):55-62.

Organización Mundial de la Salud (OMS). (2007). Salud Bucodental. Nota informativa N³18.

Philip J, Lewis R y Wysocki (2012). Patología oral y maxilofacial contemporánea. España. Editorial ELSEVIER

Quirynenm, M. (2003). A split-mouth study on periodontal and microbial parameters in children with complete unilateral cleft lip and palate. Journal of Clinical Periodontology. 2003; 30:49-56.

Vettore, M. (2011). Enfermedad periodontal y resultados adversos del embarazo: revisión de la literatura.

Parte

II.

Recuperado

en: 
https://scholar.google.com.co/scholar?q=Vettore $+\mathrm{y}+\mathrm{col}+(2011), \&$ hl=es\&as_sdt=0\&as_vis=1\&oi $=$ scholart

Zhu J, Xiao J, Liu Y, Wu J, Li JY. (2010). Caries experience in individuals with cleft lip and/or palate in China. Cleft Palate Craniofac J. 47(1):43-47. Recuperado en: https://www.ncbi.nlm.nih.gov/pubmed/20078202 